\title{
On the Connes-Kreimer construction of Hopf Algebras
}

\author{
I. Moerdijk
}

\begin{abstract}
We give a universal construction of families of Hopf $\mathbb{P}$-algebras for any Hopf operad $\mathbb{P}$. As a special case, we recover the Connes-Kreimer Hopf algebra of rooted trees.
\end{abstract}

Keywords: Hopf operad, Hopf algebra, Hochschild cohomology.

In [K], CK] a Hopf algebra $H$ of rooted trees is discussed. This algebra originates in problems of renormalisation $[\mathrm{K}]$ and is closely related to the Hopf algebra introduced in $\mathrm{CM}$ in the context of cyclic homology and foliations. The algebra $H$ is the polynomial algebra on countably many indeterminates $T$, one for each finite rooted tree $T$. Its comultiplication is given by the formula

$$
\Delta(T)=1 \otimes T+T \otimes 1+\sum_{c} F_{c} \otimes R_{c}
$$

see CK. Here $c$ ranges over all "cuts" (prunings) of the tree $T$. Such cuts are assumed non-empty, and to contain at most one edge on each branch. $R_{c}$ is the part of the tree which remains after having performed the pruning, and $F_{c}$ is the product of subtrees which have fallen on the ground. In CK it is proved that this comultiplication indeed makes $H$ into a Hopf algebra. Furthermore, $H$ is equipped with a linear endomorphism $\lambda$, which is a universal cocycle for a suitably defined Hochschild cohomology of Hopf algebras.

The first aim of this note is to show that all these properties can in fact be deduced from a more basic universal property of $H$. Namely, $H$ is the initial object in the category of (commutative unitary) algebras equipped with a linear endomorphism. Having realized that this is the case, it becomes clear that $H$ is in fact equipped with a large family of Hopf algebra structures, all making the endomorphism $\lambda$ into a universal cocycle for the corresponding Hochschild cohomology. For example, for any two complex numbers $q_{1}$ and $q_{2}$, there is a coproduct on $H$, uniquely determined by the identity

$$
\Delta(\lambda(T))=\sum q_{1}^{\left|T_{(1)}\right|} \cdot T_{(1)} \otimes \lambda\left(T_{(2)}\right)+\lambda\left(T_{(1)}\right) \otimes q_{2}^{\left|T_{(2)}\right|} \cdot T_{(2)},
$$

where $|T|$ denotes the number of nodes in the tree $T$. For $q_{1}=1$ and $q_{2}=0$ one recovers the Hopf algebra structure of CK.

The second aim is to describe how this construction applies more generally to "algebras" for any operad $\mathbb{P}$ on an additive category, as soon as one has a well-behaved tensor product of algebras. More precisely, we will show that if $\mathbb{P}$ is a "Hopf operad" on a symmetric monoidal additive category, then the initial object in the category of $\mathbb{P}$-algebras equipped with a "linear" endomorphism is naturally equipped with a family of natural Hopf $\mathbb{P}$-algebra structures. The algebra of rooted trees then becomes the extreme instance of this construction where the operad $\mathbb{P}$ is the unit object in each degree. 
Acknowledgements. My attention was first drawn to the algebra $H$ by A. Connes at the "Karoubi Fest" in Paris (November 1998). I would like to thank Ezra Getzler and André Joyal for helpful discussion. I am indebted to the Dutch Science Foundation (NWO) for financial support. The main results of this paper were first presented at the Newton Institute, in February 1999.

\section{Operads and algebras.}

1.1 The underlying category. In this preliminary section we will consider operads on a category $\mathcal{C}$. We will assume that $\mathcal{C}$ is a symmetric monoidal additive category, with countable sums and quotients of actions by finite groups on objects of $\mathcal{C}$. (In most cases, $\mathcal{C}$ will be closed under all small colimits.) As an example, the reader may wish to keep the category of vector spaces over a field $k$ in mind in what follows. We will write $k$ for the unit object of $\mathcal{C}$, and $a, l, r$ for the associativity and unit isomorphisms. The symmetry will be denoted by $c$, with components $c_{X, Y}: X \otimes Y \rightarrow Y \otimes X$. We will assume that $\otimes$ is an additive functor in each variable separately. Often, the isomorphisms $a, l, r$ will be suppressed from the notation, and we identify $k \otimes X$ with $X$, and $X \otimes(Y \otimes Z)$ with $(X \otimes Y) \otimes Z$, etc. This is justified, on the basis of Mac Lane's coherence theorem. See CWM for details.

1.2 Operads. ([M], $[\mathrm{KM}$, GK],$\ldots$ ) We will consider operads $\mathbb{P}$ on such a category $\mathcal{C}$, and write $\mathbb{P}(n)$ for the object (of $\mathcal{C}$ ) of $n$-ary operations. We will always assume that our operads have a distinguished "unit element" $u: k \rightarrow$ $\mathbb{P}(0)$. We will not assume that this map is an isomorphism, i.e. that $\mathbb{P}$ is unitary in the sense of [KM]. Many operads are unitary, but the constructions of 1.3 lead us out of unitary operads. Note that the unit $u: k \rightarrow \mathbb{P}(0)$ provides us with a unit $u_{A}: k \rightarrow A$ in any $\mathbb{P}$-algebra $A$.

The functor underlying the monad on $\mathcal{C}$ whose algebras are $\mathbb{P}$-algebras will be denoted by $F_{\mathbb{P}}: \mathcal{C} \rightarrow \mathcal{C}$; so for any object $V$ in $\mathcal{C}$,

$$
F_{\mathbb{P}}(V)=\coprod_{n \geq 0} \mathbb{P}(n) \otimes_{\Sigma_{n}} V^{\otimes n} .
$$

This object $F_{\mathbb{P}}(V)$ is the free $\mathbb{P}$-algebra generated by $V$.

1.3 Two constructions. (i) If $\mathbb{P}$ is an operad on $\mathcal{C}$ and $G$ is an object of $\mathcal{C}$, there is an operad $\mathbb{P}_{G}$ whose algebras are $\mathbb{P}$-algebras equipped with a map from $G$. Thus, $\mathbb{P}_{G}$ is obtained from $\mathbb{P}$ by adding $G$ to the space $\mathbb{P}(0)$ of "constants" (nullary operations). Explicitly,

$$
\mathbb{P}_{G}(n)=\coprod_{p \geq 0} \mathbb{P}(n+p) \otimes_{\Sigma_{p}} G^{\otimes p} .
$$

Note that the initial $\mathbb{P}_{G}$-algebra $\mathbb{P}_{G}(0)$ is the free $\mathbb{P}$-algebra $F_{\mathbb{P}}(G)$ on $G$.

(ii) Let $\mathbb{P}$ be an operad on $\mathcal{C}$. A $\mathbb{P}[t]$-algebra is a pair $(A, \alpha)$ where $A$ is a $\mathbb{P}$-algebra and $\alpha: A \rightarrow A$ is a map in $\mathcal{C}$. (We will often refer to maps in $\mathcal{C}$ as "linear maps", to contrast them with $\mathbb{P}$-algebra homomorphisms.) A map between $\mathbb{P}[t]$-algebras $(A, \alpha) \rightarrow(B, \beta)$ is a map of $\mathbb{P}$-algebras $f: A \rightarrow B$ such that $\beta f=f \alpha$. This defines a category of $\mathbb{P}[t]$-algebras. This category is the category of algebras for an operad, again denoted $\mathbb{P}[t]$. It is the operad obtained 
by freely adjoining a unary operation " $t$ " to $\mathbb{P}$. It is not difficult to give an explicit description of $\mathbb{P}[t]$ in terms of trees, analogous to constructions in $\mathrm{GK}$. We will not need such an explicit description.

1.4 Example. Let $\mathcal{C}$ be the category of vector spaces over a field $k$, and let $\mathbb{P}$ be the operad $\mathbb{P}(n)=k$. Its algebras are commutative unitary $k$-algebras, and the monad $F_{\mathbb{P}}$ associated to $\mathbb{P}$ is the symmetric algebra functor. The associated operad $\mathbb{P}[t]$ can be described as follows. The space $\mathbb{P}[t](n)$ is the vector space on rooted finite trees T, with one "output node", the root, and n "input nodes", labelled by $x_{1}, \ldots, x_{n}$. The inner nodes represent application of the new unary operation $t$. For example, the tree

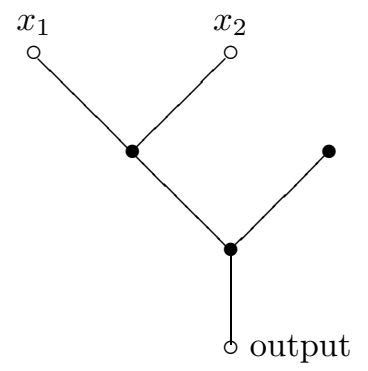

represents the binary operation $t\left(t\left(x_{1} \cdot x_{2}\right) \cdot t(1)\right)$. The tree $\circ$ consisting of just the output vertex represents the element (nullary operation) 1 . We will refer to the algebra $\mathbb{P}[t](0)$ as the algebra of finite rooted trees. It can be identified with the Connes-Kreimer algebra $H$ mentioned in the introduction. (There is a slight difference in notation, in that we have merged a product of trees into one tree with a new output node added to it.)

\section{Hopf operads.}

2.1 Coalgebras. Let $\mathcal{C}$ be a category as in 1.1. A coalgebra $\underline{X}=(X, \varepsilon, \Delta)$ is an object $X$ of $\mathcal{C}$ equipped with a coassociative comultiplication $\Delta: X \rightarrow$ $X \otimes X$, and a counit $\varepsilon: X \rightarrow k$ for this comultiplication. The associated category $\operatorname{Coalg}(\mathcal{C})$ is again a (symmetric) monoidal category, with the usual tensor product $(\underline{X} \otimes \underline{Y}$ is $X \otimes Y$ with as comultiplication the composition of $\Delta_{X} \otimes \Delta_{Y}: X \otimes Y \rightarrow(X \otimes X) \otimes(Y \otimes Y)$ and the symmetry $X \otimes c \otimes Y:$ $(X \otimes X) \otimes(Y \otimes Y) \rightarrow(X \otimes Y) \otimes(X \otimes Y))$.

2.2 Hopf operads. A Hopf operad on $\mathcal{C}$ is an operad $\mathbb{P}$ on $\mathcal{C}$ equipped with additional structure making it an operad on Coalg $(\mathcal{C})$. Thus, each $\mathbb{P}(n)$ has the structure of a coalgebra,

$$
k \stackrel{\varepsilon}{\longleftarrow} \mathbb{P}(n) \stackrel{\Delta}{\longrightarrow} \mathbb{P}(n) \otimes \mathbb{P}(n),
$$

this structure is $\Sigma_{n}$-invariant, and the structure maps of the operad $\mathbb{P}(n) \otimes$ $\mathbb{P}\left(k_{1}\right) \otimes \cdots \otimes \mathbb{P}\left(k_{n}\right) \rightarrow \mathbb{P}\left(k_{1}+\cdots+k_{n}\right)$ are coalgebra maps. The notion of a Hopf operad has been introduced in GJ]. (But beware that their coalgebras are not necessarily counital.) I will sometimes write $\mathbb{P}$ for this operad on $\operatorname{Coalg}(\mathcal{C})$, as opposed to the operad $\mathbb{P}$ on $\mathcal{C}$. The Hopf operad $\mathbb{P}$ is cocommutative if each of the coalgebras $\mathbb{P}(n)$ is. 
If $\mathbb{P}$ is a Hopf operad, then the tensor product $A \otimes B$ of two $\mathbb{P}$-algebras $A$ and $B$ is again a $\mathbb{P}$-algebra, by the maps

$$
\begin{gathered}
\mathbb{P}(n) \otimes(A \otimes B)^{\otimes n} \stackrel{\Delta \otimes \mathrm{id}}{\longrightarrow} \mathbb{P}(n) \otimes \mathbb{P}(n) \otimes(A \otimes B)^{\otimes n} \stackrel{c}{\longrightarrow} \\
\left(\mathbb{P}(n) \otimes A^{\otimes n}\right) \otimes\left(\mathbb{P}(n) \otimes B^{\otimes n}\right) \longrightarrow A \otimes B .
\end{gathered}
$$

Moreover, the counits $\varepsilon: \mathbb{P}(n) \rightarrow k$ in (11) make $k$ into a $\mathbb{P}$-algebra, which is a unit for this tensor product of $k$-algebras. Thus, the category of $\mathbb{P}$-algebras is again a monoidal category (symmetric if $\mathbb{P}$ is cocommutative). A coalgebra in this category of $\mathbb{P}$-algebras is the same thing as a $\mathbb{P}$-algebra in the category Coalg $(\mathcal{C})$ of coalgebras, and (as in $G$. $]$ ) will be referred to as a Hopf $\mathbb{P}$-algebra.

2.3 Example. The free $\mathbb{P}$-algebra $F_{\mathbb{P}}(G)$ on an object $G$ has a canonical Hopf $\mathbb{P}$-algebra structure, cocommutative if $\mathbb{P}$ is. Indeed, since $F_{\mathbb{P}}(G)$ is free, the maps $0: G \rightarrow k$ and id $\otimes 1+1 \otimes \mathrm{id}: G \rightarrow F_{\mathbb{P}}(G) \otimes F_{\mathbb{P}}(G)$ into $\mathbb{P}$-algebras extend uniquely to $\mathbb{P}$-algebra maps

$$
k \stackrel{\varepsilon}{\longleftarrow} F_{\mathbb{P}}(G) \stackrel{\Delta}{\longrightarrow} F_{\mathbb{P}}(G) \otimes F_{\mathbb{P}}(G),
$$

and one easily checks that this provides the claimed structure.

\section{The Connes-Kreimer construction.}

Let $\mathbb{P}$ be a Hopf operad on a category $\mathcal{C}$ as before, and let $\mathbb{P}[t]$ be the associated operad whose algebras are $\mathbb{P}$-algebras equipped with a "linear" endomorphism. We now present a general construction of Hopf $\mathbb{P}$-algebras, of which the ConnesKreimer Hopf algebra is a special case.

3.1 The initial $\mathbb{P}[t]$-algebra. Let $(H, \lambda)$ denote the initial $\mathbb{P}[t]$-algebra, i.e. $(H, \lambda)=\mathbb{P}[t](0)$. Thus $H$ is a $\mathbb{P}$-algebra, $\lambda: H \rightarrow H$ is a linear map (i.e. just an arrow in $\mathcal{C}$ ), and these have the following universal property: For any $\mathbb{P}$-algebra $A$ and any linear map $\alpha: A \rightarrow A$, there is a unique $\mathbb{P}$-algebra map $\varphi: H \rightarrow A$ such that $\alpha \varphi=\varphi \lambda$.

3.2 Lemma. There is a unique augmentation $\varepsilon: H \rightarrow k$ with $\lambda \varepsilon=0$.

Proof: Apply the universal property to the $\mathbb{P}$-algebra $k$ with the zero endomorphism.

Next, let $\sigma_{1}, \sigma_{2}: H \rightarrow H$ be two linear maps. Let

$$
\left(\sigma_{1}, \sigma_{2}\right)=\sigma_{1} \otimes \lambda+\lambda \otimes \sigma_{2}: H \otimes H \rightarrow H \otimes H .
$$

This gives $H \otimes H$ the structure of a $\mathbb{P}[t]$-algebra. So there is a unique $\mathbb{P}$-algebra map

$$
\Delta=\Delta_{\sigma_{1}, \sigma_{2}}: H \rightarrow H \otimes H
$$

such that $\left(\sigma_{1}, \sigma_{2}\right) \circ \Delta=\Delta \circ \lambda$. 
3.3 Lemma. (i) If $\varepsilon \sigma_{i}=\varepsilon$ for $i=1,2$ then $\varepsilon: H \rightarrow k$ is a counit for $\Delta$.

(ii) If, in addition, $\Delta \sigma_{i}=\left(\sigma_{i} \otimes \sigma_{i}\right) \Delta$ for $i=1,2$ then $\Delta$ is coassociative.

Proof: (i) Consider the maps

$$
(H, \lambda) \stackrel{\Delta}{\longrightarrow}\left(H \otimes H,\left(\sigma_{1}, \sigma_{2}\right)\right) \underset{\varepsilon \otimes \mathrm{id}}{\stackrel{\mathrm{id} \otimes \varepsilon}{\longrightarrow}}(H, \lambda),
$$

where on the right the isomorphisms $H \otimes k=H=k \otimes H$ have been suppressed. By initiality of $H$, it is enough to prove that id $\otimes \varepsilon$ and $\varepsilon \otimes$ id are $\mathbb{P}[t]$-homomorphisms. This is indeed the case, since

$$
\begin{aligned}
(\mathrm{id} \otimes \varepsilon)\left(\sigma_{1}, \sigma_{2}\right) & =(\mathrm{id} \otimes \varepsilon)\left(\sigma_{1} \otimes \lambda+\lambda \otimes \sigma_{2}\right) \quad \text { (definition) } \\
& =\sigma_{1} \otimes \varepsilon \lambda+\lambda \otimes \varepsilon \sigma_{2} \\
& =\lambda \otimes \varepsilon \sigma_{2} \quad(\varepsilon \lambda=0) \\
& =\lambda \otimes \varepsilon \quad \text { (assumption) } \\
& =\lambda \circ(\mathrm{id} \otimes \varepsilon),
\end{aligned}
$$

and similarly $(\varepsilon \otimes \mathrm{id})\left(\sigma_{1}, \sigma_{2}\right)=\lambda \circ(\varepsilon \otimes \mathrm{id})$.

(ii) Consider the map $\nu: H \otimes H \otimes H \rightarrow H \otimes H \otimes H$,

$$
\nu=\lambda \otimes \sigma_{2} \otimes \sigma_{2}+\sigma_{1} \otimes \lambda \otimes \sigma_{2}+\sigma_{1} \otimes \sigma_{1} \otimes \lambda .
$$

This makes $H^{\otimes 3}$ into a $\mathbb{P}[t]$-algebra, so there is a unique $\mathbb{P}[t]$-homomorphism $(H, \lambda) \rightarrow\left(H^{\otimes 3}, \nu\right)$. It thus suffices to show that (id $\left.\otimes \Delta\right) \Delta$ and $(\Delta \otimes$ id $) \Delta$ both are. For the first,

$$
\begin{aligned}
(\mathrm{id} \otimes \Delta) \Delta \lambda & =(\mathrm{id} \otimes \Delta)\left(\sigma_{1} \otimes \lambda+\lambda \otimes \sigma_{2}\right) \Delta \\
& =\left(\sigma_{1} \otimes \Delta \lambda+\lambda \otimes \Delta \sigma_{2}\right) \Delta \\
& =\left(\sigma_{1} \otimes \sigma_{1} \otimes \lambda+\sigma_{1} \otimes \lambda \otimes \sigma_{2}+\lambda \otimes \sigma_{2} \otimes \sigma_{2}\right)(\mathrm{id} \otimes \Delta) \Delta \\
& =\nu(\mathrm{id} \otimes \Delta) \Delta .
\end{aligned}
$$

The calculation for $(\Delta \otimes \mathrm{id}) \Delta$ is similar.

The preceding lemmas prove:

3.4 Theorem. The initial $\mathbb{P}[t]$-algebra $(H, \lambda)$ has a natural family of Hopf $\mathbb{P}$-algebra structures, parametrized by pairs $\sigma_{1}, \sigma_{2}: H \rightarrow H$ satisfying the conditions of Lemma 3.3.

3.5 Example. The conditions of Lemma 3.3 are always satisfied if one takes $\sigma_{i}$ to be the identity $H \rightarrow H$ or the composition of the counit $\varepsilon: H \rightarrow k$ and the unit $u: k \rightarrow H$, or any convex combination $\alpha \cdot \mathrm{id}+\beta \cdot u \varepsilon: H \rightarrow H$ of these two (for $\alpha, \beta: k \rightarrow k$ with $\alpha+\beta=$ id). This provides many different Hopf $\mathbb{P}$-algebra structures on $H$.

3.6 Example. Consider again the case of the commutative unitary algebra operad of 1.4. Then $H$ is the algebra of finite rooted trees $T$. Note that $\varepsilon(T)=0$ as soon as $T$ has at least one inner node. Write $|T|$ for the number of inner nodes of $T$. Now let $q_{1}, q_{2} \in k$ be any two numbers, and let

$$
\sigma_{i}=q_{i}^{|T|} \cdot T, \quad \text { for } i=1,2
$$


Then $\sigma_{1}$ and $\sigma_{2}$ satisfy the condition of Lemma 3.3. Thus for any two $q_{1}, q_{2} \in k$, the algebra $H$ has a Hopf algebra structure, with the usual counit, and with comultiplication completely determined by the identity

$$
\Delta \lambda(T)=\sum q_{1}^{\left|T_{(1)}\right|} T_{(1)} \otimes \lambda\left(T_{(2)}\right)+\lambda\left(T_{(1)}\right) \otimes q_{2}^{\left|T_{(2)}\right|} \cdot T_{(2)}
$$

where we write $\Delta(T)=\sum T_{(1)} \otimes T_{(2)}$ as usual [B]. For the values $q_{1}=1$ and $q_{2}=0$ one finds $\sigma_{1}=$ id and $\sigma_{2}=\varepsilon$, and one recovers the Hopf algebra structure of [CK].

3.7 Remark. The results and examples in this section have been stated for the initial $\mathbb{P}[t]$-algebra $(H, \lambda)=\mathbb{P}[t](0)$. Similar facts hold for the free $\mathbb{P}[t]$-algebra generated by any object $G$ of $\mathcal{C}$. Writing $(H[G], \lambda)$ for this algebra and $j: G \rightarrow$ $H[G]$ for the universal map from $G$, one defines $\Delta: H[G] \rightarrow H[G] \otimes H[G]$ from $\sigma_{1}$ and $\sigma_{2}$ as the unique map of $\mathbb{P}[t]$-algebras satisfying $\Delta \lambda=\left(\sigma_{1} \otimes \lambda+\lambda \otimes \sigma_{2}\right) \Delta$ as before and extending the map $u \otimes j+j \otimes u: G \rightarrow H[G] \otimes H[G]$ (where $u: k \rightarrow$ $H[G]$ is the unit). However, rather than doing the calculation again, this can be seen as a formal consequence of the statements made for the initial algebra, because the free $\mathbb{P}[t]$-algebra on $G$ is the initial $\mathbb{P}_{G}[t]$-algebra (cf. 1.3.(i)), and $\mathbb{P}_{G}$ is a Hopf operad whenever $\mathbb{P}$ is.

\section{Hochschild cohomology.}

In CK it is proved that for the Connes-Kreimer algebra $(H, \lambda)$ (cf. Example 3.6), the map $\lambda$ is a universal 1-cocycle for Hochschild cohomology. In this section, we show that this result extends to our more general construction.

Recall the definition of the Hochschild cohomology groups $H^{*}(A, M)$ for any algebra $A$ and any bimodule $M$, from the complex with maps $A^{\otimes n} \rightarrow M$ as cochains (see e.g. [4, formula (1.5.1.1)]). Turning around all the arrows in a diagrammatic form of this definition, one obtains a cohomology $H^{*}(E, C)$ of a coalgebra $C$ with coefficients in a bicomodule $E$, as the cohomology of the complex $C^{n}(E, C)=\operatorname{Hom}_{\mathcal{C}}\left(E, C^{\otimes n}\right)$. Explicitly, this is the cohomology of the simplicial abelian group with the face maps $d_{i}: C^{n-1}(E, C) \rightarrow C^{n}(E, C)$ defined for $\varphi: E \rightarrow C^{\otimes(n-1)}$ by

$$
d_{i}(\varphi)= \begin{cases}E \stackrel{l}{\longrightarrow} C \otimes E \stackrel{C \otimes \varphi}{\longrightarrow} C \otimes C^{\otimes n-1}=C^{\otimes n} & (i=0) \\ E \stackrel{\varphi}{\longrightarrow} C^{\otimes n-1} \stackrel{\Delta(i)}{\longrightarrow} C^{\otimes n} & (0<i<n) \\ E \stackrel{r}{\longrightarrow} E \otimes C \stackrel{\varphi \otimes C}{\longrightarrow} C^{\otimes n} & (i=n) .\end{cases}
$$

Here $l$ and $r$ are the left and right coactions, and $\Delta^{(i)}=C^{\otimes(i-1)} \otimes \Delta \otimes C^{\otimes(n-i-1)}$. Note that this cohomology $H^{*}(E, C)$ is contravariant in $E$ and covariant in $C$.

In particular, given "linear" maps $\sigma_{1}, \sigma_{2}: C \rightarrow C$, we can view $C$ itself as a $C$-bimodule $\sigma_{1} C_{\sigma_{2}}$, with left action $C \stackrel{\Delta}{\longrightarrow} C \otimes C \stackrel{\sigma_{1} \otimes C}{\longrightarrow} C \otimes C$ and right action $C \stackrel{\Delta}{\longrightarrow} C \otimes C \stackrel{C \otimes \sigma_{2}}{\longrightarrow} C \otimes C$. We denote the corresponding cohomology by

$$
H H_{\sigma_{1}, \sigma_{2}}^{*}(C) \text {. }
$$

A map $\varphi: C \rightarrow C$ is a 1-cocycle for this cohomology precisely when

$$
\Delta \circ \varphi=\left(\sigma_{1} \otimes \varphi+\varphi \otimes \sigma_{2}\right) \Delta .
$$


Now let us go back to the context of a Hopf operad $\mathbb{P}$ on our underlying category $\mathcal{C}$.

4.1 Natural twisting functions. Call $\sigma$ a natural twisting function if $\sigma$ assigns to each Hopf $\mathbb{P}$-algebra $C$ a linear endomorphism $\sigma=\sigma^{(C)}: C \rightarrow C$, which is natural for morphisms of augmented $\mathbb{P}$-algebras (i.e. if $f: C \rightarrow D$ is such a morphism then $\left.f \circ \sigma^{(C)}=\sigma^{(D)} \circ f\right)$, and has the property that $\sigma^{(k)}$ is the identity. Note that this implies that $\varepsilon \circ \sigma^{(C)}=\varepsilon$. For example, the identity $C \rightarrow C$ and the composition $C \stackrel{\varepsilon}{\longrightarrow} k \stackrel{u}{\longrightarrow} C$ of the augmentation and the unit are natural twisting functions, as is any convex combination $\alpha \cdot \mathrm{id}+\beta \cdot u \varepsilon: C \rightarrow C$ of these two (for $\alpha, \beta: k \rightarrow k$ with $\alpha+\beta=$ id).

Now let $(H, \lambda)$ be the initial $\mathbb{P}[t]$-algebra, and let $\sigma_{1}=\sigma_{1}^{(H)}, \sigma_{2}=\sigma_{2}^{(H)}$ : $H \rightarrow H$ be the components of two natural twisting functions. Suppose that $\sigma_{1}$ and $\sigma_{2}$ define a Hopf $\mathbb{P}$-algebra structure $(H, \Delta, \varepsilon)$ on $H$, by Theorem 3.4. Observe that the defining equation $\left(\sigma_{1}, \sigma_{2}\right) \Delta=\Delta \lambda$ for the coproduct states precisely that $\lambda$ is a 1-cocycle for $H H_{\sigma_{1}, \sigma_{2}}^{*}(H)$. The following theorem is now a consequence of the universal property $(3.1)$ of $(H, \lambda)$.

4.2 Theorem. The map $\lambda$ is the universal 1-cocycle. More explicitly, if $B$ is a Hopf $\mathbb{P}$-algebra and $\gamma$ is a 1-cocycle in the complex defining $H_{\sigma_{1}, \sigma_{2}}^{*}(B)$, there is a unique Hopf $\mathbb{P}$-algebra map $c_{\gamma}: H \rightarrow B$ such that $c_{\gamma} \circ \lambda=\gamma \circ c_{\gamma}$.

Proof: By the universal property of $H$ and $\lambda$, there is a unique $\mathbb{P}$-algebra map $c=c_{\gamma}: H \rightarrow B$ such that $\gamma c=c \lambda$. It suffices to show that $c$ is a coalgebra map. First, we show that $c$ is a map of augmented algebras, i.e. $\varepsilon \circ c=\varepsilon$. By initiality of $(H, \lambda)$, it suffices to show that the composite $(H, \lambda) \stackrel{c}{\longrightarrow}(B, \gamma) \stackrel{\varepsilon}{\longrightarrow}(k, 0)$ is a map of $\mathbb{P}[t]$-algebras; in other words, that $\varepsilon \gamma=0$. To prove this, apply $\varepsilon \otimes \varepsilon$ to the cocycle condition $\Delta \gamma=\left(\sigma_{1} \otimes \gamma+\gamma \otimes \sigma_{2}\right) \Delta$. Using that $(\varepsilon \otimes \varepsilon) \Delta=\varepsilon$, and $\varepsilon \sigma_{i}=\varepsilon$ (as observed above), this yields $\varepsilon \gamma=(\varepsilon \otimes \varepsilon \gamma+\varepsilon \gamma \otimes \varepsilon) \Delta=\varepsilon \gamma+\varepsilon \gamma$. Thus $\varepsilon \gamma=0$, as desired.

Next, we show that the map $c$ preserves coproducts. Observe that, by initiality of $(H, \lambda)$, the square

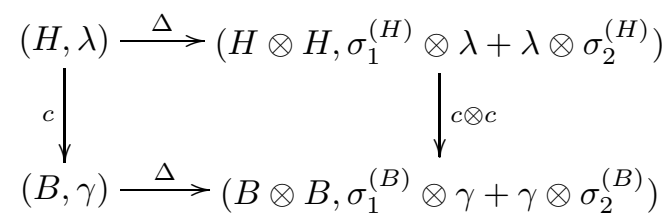

necesarily commutes as soon as all four maps are $\mathbb{P}[t]$-algebra homomorphisms. The map $c \otimes c$ is the only one for which this still has to be shown. But, we have just proved that $c$ is a map of augmented $\mathbb{P}$-algebras, so $c \circ \sigma_{i}^{(H)}=\sigma_{i}^{(B)} \circ c$ by naturality. Since alse $c \lambda=\gamma c$, the map $c \otimes c$ is indeed a map of $\mathbb{P}[t]$-algebras. This completes the proof of the theorem.

\section{Remarks on functoriality.}

We continue to work in the context of Hopf operads on a category $\mathcal{C}$ as in 1.1. 
5.1 Adjoint functors. Let $\varphi: \mathbb{Q} \rightarrow \mathbb{P}$ be a map of Hopf operads. Then $\varphi$ induces functors $\varphi^{*}:(\mathbb{P}$-algebras $) \rightarrow(\mathbb{Q}$-algebras $)$ and $\bar{\varphi}^{*}:($ Hopf $\mathbb{P}$-algebras $) \rightarrow$ (Hopf $\mathbb{Q}$-algebras). Also, $\varphi$ gives a functor $\varphi^{*}:(\mathbb{P}[t]$-algebras $) \rightarrow(\mathbb{Q}[t]$-algebras $)$, by $\varphi^{*}(B, \beta)=\left(\varphi^{*}(B), \beta\right)$. If the relevant coequalizers exists in $\mathcal{C}$ then the first

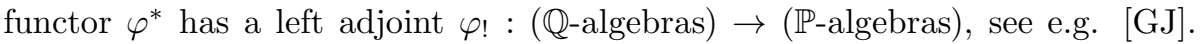
Note that $\varphi^{*}(k)=k$ and that the (first) functor $\varphi^{*}$ commutes with tensor products of algebras. Hence by adjointness, there are canonical maps of $\mathbb{P}$-algebras $\varphi_{!}(k) \rightarrow k$ and $\varphi_{!}(A \otimes B) \rightarrow \varphi_{!}(A) \otimes \varphi_{!}(B)$. Using these maps, one obtains a lifting of $\varphi$ ! to a left adjoint $\bar{\varphi}_{!}:\left(\right.$Hopf- $\mathbb{P}$-algebras) $\rightarrow$ (Hopf- $\mathbb{Q}$-algebras) for $\bar{\varphi}^{*}$.

Now let $(H, \lambda)$ be the initial $\mathbb{P}[t]$-algebra and $(K, \mu)$ the one for $\mathbb{Q}$. Let $j_{0}:(K, \mu) \rightarrow\left(\varphi^{*}(H), \lambda\right)$ be the unique map of $\mathbb{Q}[t]$-algebras, and note that this is a map of augmented $\mathbb{Q}$-algebras. Let $j: \varphi_{!}(K) \rightarrow H$ be the adjoint map; this is a map of augmented $\mathbb{P}$-algebras. Next, consider natural twisting functions $\sigma_{1}, \sigma_{2}$ on $\mathbb{Q}$-algebras. These also induce $\sigma_{i}: H \rightarrow H$ on any $\mathbb{P}$-algebra $H$, by $\sigma_{i}=\sigma_{i}^{\left(\varphi^{*}(H)\right)}$.

5.2 Proposition. Suppose $\sigma_{1}$ and $\sigma_{2}$ satisfy the conditions of Theorem 3.4 so as to make $H$ and $K$ into Hopf $\mathbb{P}$-(respectively $\mathbb{Q}$-)algebras. Then $j_{0}: K \rightarrow$ $\varphi^{*}(H)$ and $j: \varphi_{!}(K) \rightarrow H$ are maps of Hopf $\mathbb{P}$-(resp. $\mathbb{Q}$-)algebras.

Proof: The second assertion for $j$ follows from the first for $j_{0}$ by adjointness. To see that the map $j_{0}$ preserves the coproduct, simply apply initiality of $(K, \mu)$ to the square

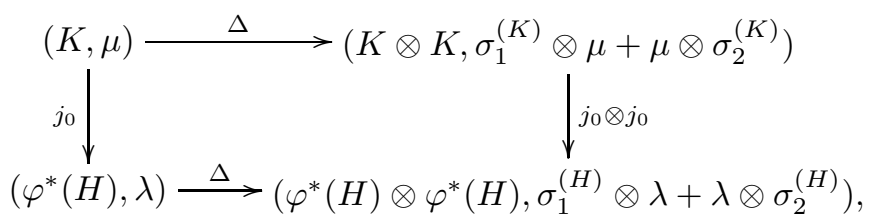

exactly as in the proof of Theorem 4.2.

5.3 The operad $\mathbb{B}$. A pointed object is an object $X$ of $\mathcal{C}$ equipped with a "basepoint" $u: k \rightarrow X$. We call $X$ well-pointed if $X$ is equipped with an augmentation $\varepsilon: X \rightarrow k$ with $\varepsilon u=$ id. Such an object splits as $X=k \oplus \tilde{X}$ where $\tilde{X}=\operatorname{Ker}(\varepsilon)$. Let $\mathbb{B}$ be the operad whose algebras are pointed objects. If $\mathbb{P}$ is any (Hopf) operad then the unit of $\mathbb{P}$ gives a map of operads $u: \mathbb{B} \rightarrow \mathbb{P}$. We consider the left adjoint $u$ ! of the induced functor $u^{*}:(\mathbb{P}$-algebras $) \rightarrow$ (B-algebras).

5.4 Lemma. If $X$ is well-pointed then $u_{!}(X)=F_{\mathbb{P}}(\tilde{X})$, the free $\mathbb{P}$-algebra on $\tilde{X}$.

Proof: $\quad$ Let $k \stackrel{u}{\longrightarrow} X \stackrel{\varepsilon}{\longrightarrow} k$ be a well-pointed object. Let $w: X \rightarrow F_{\mathbb{P}}(\tilde{X})=$ $F(\tilde{X})$ be the map $k \oplus \tilde{X} \rightarrow F(\tilde{X})$ obtained from the unit $u_{F(\tilde{X})}: k \rightarrow F(\tilde{X})$ of this free algebra together with the canonical map $\mu: \tilde{X} \rightarrow F(\tilde{X})$. We claim that $w$ is the universal base-point preserving map from $X$ into a $\mathbb{P}$-algebra. Indeed, suppose $f: X \rightarrow A$ is any map into the underlying object $A$ of a $\mathbb{P}$-algebra $\underline{A}$, with $f \circ u=u_{\underline{A}}$. Since $F(\tilde{X})$ is the free algebra, the restriction $f \uparrow \tilde{X}: \tilde{X} \rightarrow A$ 
extends uniquely to a $\mathbb{P}$-algebra map $\underline{f}: F(\tilde{X}) \rightarrow \underline{A}$. It is easy to check that $\underline{f} \circ w=f$ for this map $\underline{f}$.

Now let $(A, \alpha)$ be the initial $\mathbb{B}[t]$-algebra, and $(H, \lambda)$ the initial $\mathbb{P}[t]$-algebra as before. Let $\sigma_{1}, \sigma_{2}$ be natural twisting functions on $\mathbb{B}$-algebras. Suppose $\sigma_{1}^{(A)}, \sigma_{2}^{(A)}: A \rightarrow A$ define a Hopf algebra structure on $A$, and $\sigma_{1}^{(H)}, \sigma_{2}^{(H)}: H \rightarrow$ $H$ one on $H$, by Theorem 3.4.

\subsection{Proposition. There is a canonical retraction}

$$
u_{!}(A) \underset{r}{\stackrel{j}{\rightleftarrows}} H, \quad r \circ j=\mathrm{id},
$$

where $j$ is a map of Hopf $\mathbb{P}$-algebras and $r$ one of augmented $\mathbb{P}$-algebras.

Proof: The map $j: u_{!}(A) \rightarrow H$ is the one of Proposition 5.2. The map $r: H \rightarrow u_{!}(A)$ is the unique map $(H, \lambda) \rightarrow\left(u_{!}(A), \bar{\alpha}\right)$ of $\mathbb{P}[t]$-algebras, for the map $\bar{\alpha}$ defined as follows. Since $A$ has an augmentation $\varepsilon$ with $\varepsilon \alpha=0$ (Lemma 3.2 ), we can write $A=k \oplus \tilde{A}$ where $\alpha$ maps $A$ into $\tilde{A}$. Also, the free $\mathbb{P}$-algebra $u_{!}(A)=F_{\mathbb{P}}(\tilde{A})$, briefly $F(\tilde{A})$, is augmented, hence splits as $u_{!}(A)=k \oplus F(\tilde{A})^{\sim}$. Now define $\bar{\alpha}$ on these two summands separately: on $k$ it is the composition

$$
k \stackrel{u}{\longrightarrow} A \stackrel{\alpha}{\longrightarrow} \tilde{A} \rightarrow F(\tilde{A})
$$

and on the other summand it is the map

$$
F(\tilde{A})^{\sim} \subseteq F(\tilde{A}) \stackrel{F(\tilde{\alpha})}{\longrightarrow} F(\tilde{A})
$$

where $\tilde{\alpha}: \tilde{A} \rightarrow \tilde{A}$ is the restriction of $\alpha$. Note that the map $\bar{\alpha}$ thus defined satisfies the identities

$$
\bar{\alpha} w=w \alpha, \varepsilon \bar{\alpha}=0,
$$

where $w: A \rightarrow u_{!}(A)$ is the universal map as in the proof of the previous lemma.

We claim that $r \circ j=$ id. By adjointness, it suffices to show $r j w=w$ as maps of pointed objects. Now $w \alpha=\bar{\alpha} w$ as we have seen. Also, $j: u_{!}(A) \rightarrow H$ is obtained from $j_{0}: A \rightarrow u^{*}(H)$ by adjointness, hence $j w=j_{0}$. Thus $(r j w) \alpha=$ $r j_{0} \alpha=r \lambda j_{0}=\bar{\alpha} r j_{0}=\bar{\alpha}(r j w)$. This shows that $w$ and $r j w$ are both maps of $\mathbb{B}[t]$-algebras on $(A, \alpha)$, hence equal by initiality.

It remains to observe that $r$ respects the augmentation. Since $r:(H, \lambda) \rightarrow$ $\left(u_{!}(A), \bar{\alpha}\right)$ and $\varepsilon:\left(u_{!}(A), \bar{\alpha}\right) \rightarrow(k, 0)$ are both maps of $\mathbb{P}[t]$-algebras, so is the composite $\varepsilon r$. So $\varepsilon r=\varepsilon$ by initiality of $(H, \lambda)$. This shows that $r$ preserves the augmentation, and completes the proof.

5.6 Example. Let $(H, \lambda)$ be the Connes-Kreimer Hopf algebra of Example 3.6. For the same twisting functions $\sigma_{1}=\mathrm{id}$ and $\sigma_{2}=u \varepsilon$, the initial $\mathbb{B}[t]$-algebra $(A, \alpha)$ is the vector space with basis $x_{0}, x_{1}, x_{2}, \ldots$, where $x_{0}$ is the base point and $\alpha\left(x_{n}\right)=x_{n+1}$. Thus $u_{!}(A)$ is the algebra $k\left[x_{1}, x_{2}, \ldots\right]$, where we identify $x_{0}$ with $1 \in u_{!}(A)$. The Hopf algebra structure is given by $\Delta\left(x_{n}\right)=\sum_{i=0}^{n} x_{i} \otimes x_{n-i}$. The embedding $j$ identifies $u_{!}(A)$ with the subalgebra of "linear trees" of $H$ 
(considered also in $\overline{\mathrm{CK}}]$ ), and $x_{n}$ with $\lambda^{n}(1) \in H$. The retraction $r: H \rightarrow u_{!}(A)$ sends a tree $T$ to the product of all the maximal branches through $T$. For example, the tree

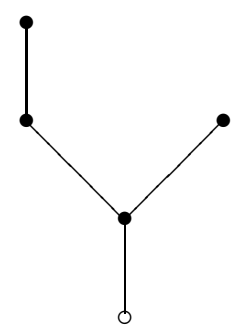

representing $\lambda\left(\lambda^{2}(1) \cdot \lambda(1)\right)$ is sent to $x_{3} \cdot x_{1}$. Note that $r$ does not commute with coproducts.

\section{References}

[CK] A. Connes, D. Kreimer, Hopf algebras, renormalization and noncommutative geometry, Comm. Math. Phys., 199 (1998), 203-242.

[CM] A. Connes, H. Moscovici, Hopf algebras, cyclic homology and the transverse index theorem, Comm. Math. Phys., 198 (1998), 199-246.

[GJ] E. Getzler, J. Jones, Operads, homotopy algebra and iterated integrals for double loop spaces, Preprint, 1992.

[GK] V.A. Ginzburg, M.M. Kapranov, Koszul duality for operads, Duke Math. J., 76 (1994), 203-272.

[K] D. Kreimer, On the Hopf algebra structure of perturbative quantum field theories, Adv. Theo. Math. Phys. 2.2 (1998), 303-334.

[KM] I. Kriz, J.P. May, Operads, algebras, modules and motives, Astérisque 233 (1995).

[L] J.-L. Loday, Cyclic Homology, Springer-Verlag, 1992.

[CWM] S. Mac Lane, Categories for the Working Mathematician, SpringerVerlag, 1971.

[M] J.P. May, The geometry of iterated loop spaces, Lecture Notes in Math. 271, Springer-Verlag, 1972.

[S] M. Sweedler, Hopf Algebras, Benjamin, New York, 1964.

Utrecht, April 1999. 